\title{
Excessive Daytime Sleepiness Is Associated with Changes in Salivary Inflammatory Genes Transcripts
}

\author{
Matthew S. Thimgan, ${ }^{1,2}$ Cristina Toedebusch, ${ }^{3}$ Jennifer McLeland, ${ }^{3}$ \\ Stephen P. Duntley, ${ }^{3,4}$ and Paul J. Shaw ${ }^{1}$ \\ ${ }^{1}$ Department of Anatomy and Physiology, Washington University School of Medicine, St. Louis, MO 63110, USA \\ ${ }^{2}$ Department of Biological Sciences, Missouri University of Science and Technology, 400 W. 11th Street, Rolla, MO 65401, USA \\ ${ }^{3}$ Department of Neurology, Washington University School of Medicine, St. Louis, MO 63110, USA \\ ${ }^{4}$ Critical Care, Pulmonary and Sleep Associates, 274 Union Boulevard, Suite 110, Lakewood, CO 80228, USA
}

Correspondence should be addressed to Paul J. Shaw; shawp@pcg.wustl.edu

Received 22 September 2014; Revised 19 January 2015; Accepted 28 January 2015

Academic Editor: Jean Louis Pepin

Copyright (C) 2015 Matthew S. Thimgan et al. This is an open access article distributed under the Creative Commons Attribution License, which permits unrestricted use, distribution, and reproduction in any medium, provided the original work is properly cited.

\begin{abstract}
Excessive daytime sleepiness (EDS) is a ubiquitous problem that affects public health and safety. A test that can reliably identify individuals that suffer from EDS is needed. In contrast to other methods, salivary biomarkers are an objective, inexpensive, and noninvasive method to identify individuals with inadequate sleep. Although we have previously shown that inflammatory genes are elevated in saliva samples taken from sleep deprived individuals, it is unclear if inflammatory genes will be elevated in clinical populations with EDS. In this study, salivary samples from individuals with sleep apnea were evaluated using the Taqman low density inflammation array. Transcript levels for 3 genes, including prostaglandin-endoperoxide synthase 2 (PTGS2), were elevated in patients with sleep apnea. Interestingly, PTGS2 was also elevated in patients with EDS but who did not have sleep apnea. These data demonstrate the feasibility of using salivary transcript levels to identify individuals that self-report excessive daytime sleepiness.
\end{abstract}

\section{Introduction}

Inadequate sleep is a pervasive problem in today's society. Insufficient sleep leads to decreased cognitive performance [1], increased sleepiness [2-4], reduced productivity [5], and increased traffic accidents [6]. Moreover, inadequate sleep increases the susceptibility of individuals to adverse health outcomes, including cardiovascular deficits [7], increased immune challenges $[8,9]$, longer recovery times after injury [10], increases in sympathetic tone [11, 12], and reduced lifespan [7]. Given the number and severity of consequences that accompany inadequate sleep, it would be helpful to have a simple and reliable test to identify vulnerable individuals before they experience adverse consequences.

One approach to accomplish this objective has been to test candidate biomarkers to determine whether they are consistently altered in subjects with inadequate or insufficient sleep. Biomarkers are objective, often endogenous factors that report changes in body chemistry and correlate with either disease state or the severity of the disease. Several biomarkers, including eyelid closures [13] and balance [14], as well as biochemical markers from blood $[15,16]$, cerebral spinal fluid [17-19], and breath analytes [20] have been evaluated as candidate biomarkers of disrupted sleep. Although each of these approaches has had limited success, assessment tools that can be used in real-world settings are not yet widely available. With that in mind, we have hypothesized that saliva, as a rich source of analytes, can be mined to identify biomarkers of sleepiness or inadequate sleep. Saliva is particularly well suited for monitoring sleepiness since it is a readily accessible biological fluid that can be easily collected using noninvasive procedures. Indeed, we have shown that transcripts for $\alpha$-amylase, Filamin- $A$, maleic enzyme, integrin, $\alpha M$, and integrin, $\alpha 5$, are all elevated in saliva samples following sleep deprivation [21-24]. Increased levels of salivary $\alpha$-amylase activity have also been shown to correlate with increased sleepiness and decreased cognitive performance in an independent study [25]. However, since 
endogenous factors are frequently modulated by a variety of physiological conditions (stress, circadian time, etc.), test of sleepiness should be comprised of a panel of independent analytes. Several studies have found that serum markers of inflammation are elevated in populations of individuals with sleep disorders [9]. For example, serum levels of interleukin 6 (IL-6) [26], interleukin 8 (IL-8) [27, 28], tumor necrosis factor- $\alpha$ (TNF- $\alpha$ ) [29], C-reactive protein (CRP) [30], intracellular adhesion molecule (ICAM) [31], selectins [32], and vascular cell adhesion molecule (VCAM) [31] have all been shown to increase in multiple populations of patients with sleep apnea. Therefore, we wanted to determine if levels of salivary inflammation transcripts could be used to identify sleepiness in a clinical population, individuals diagnosed with sleep apnea.

\section{Materials and Methods}

2.1. Subjects and Samples. 8 controls, 14 patients confirmed to have sleep apnea, and 18 patients that were suspected to have sleep apnea during their initial screen but did not exhibit sleep apnea during their overnight assessment (sleepy) were evaluated for salivary transcript levels. Both the sleep apnea group and the "sleepy" group were referred to the Washington University Sleep Laboratory due to excessive sleepiness. Samples were taken from patients at $\sim 9 \mathrm{pm}$ after they had arrived at the sleep lab and prior to beginning polysomnography. The apnea/hypopnea index (AHI) was determined using polysomnography (PSG) as previously described [33]. Subjects were free from psychiatric disorders and prescription medicines [24]. Each subject was administered the Epworth Sleepiness Scale (ESS) and their body mass index (BMI) was calculated. BMI was unavailable for one subject in the "sleepy" group. Consent was received from all participants and all protocols were approved by the Washington University School of Medicine Institutional Review Board.

2.2. Low Density Arrays. Saliva was taken and cDNA was generated according to the protocol previously described [24]. Briefly, saliva was obtained when subjects chewed on a salivette (Sarstedt, Newton, NC). One mL of RNAlater (Life Technologies, Grand Island, NY) was added to the salivette and immediately frozen on dry ice and stored at $-80^{\circ} \mathrm{C}$. At the time of transcript analysis, salivettes were thawed and the saliva-RNAlater extracts were extracted by centrifugation. RNA was purified from cell-free lysates and reversetranscribed using Superscript III (Life Technologies, Grand Island, NY) according to the manufacturer's instructions. Equal amounts of cDNA were loaded into an inflammation Taqman low density array (LDA) (Life Technologies, Grand Island, NY) and Taqman based qPCR was carried out using the 7600 real-time PCR system (Applied Biosystems, Foster City, CA). The LDAs were normalized to 18S RNA. Fold changes were determined using the method described in [34], known as the comparative $C_{T}$ method. Transcript levels for the gene of interest were first normalized within subject to $18 S$ RNA.
TABLE 1: Demographics for controls and sleep apnea.

\begin{tabular}{lcc}
\hline & Controls $(n=8)$ & OSA $(n=14)$ \\
\hline Gender & $5 \mathrm{M}, 3 \mathrm{~F}$ & $11 \mathrm{M}, 3 \mathrm{~F}$ \\
Age & $40.8 \pm 8.9$ & $49.0 \pm 12.2$ \\
AHI & - & $48.5 \pm 23.6$ \\
BMI $^{*}$ & $25.7 \pm 4.4$ & $34.6 \pm 7.1$ \\
ESS $^{*}$ & $3.9 \pm 2.7$ & $10.8 \pm 4.3$ \\
\hline
\end{tabular}

Ave. \pm Std. Dev.; ${ }^{*} P<0.05$.

2.3. Statistics. All demographic data is presented as average \pm standard deviation. For transcript analysis, if a transcript was not detected in more than $77 \%$ of the group, the transcript was deemed undetectable and not analyzed. For all detectable genes, transcript levels for each subject were determined as a fold change from the average levels of the control group. Fold changes were converted to a $\log _{2}$ scale. From the log data, average and standard error were calculated. Groups of interest were compared to control levels using a two-tailed, unpaired Student's $t$-test and the significance level was set at $0.05 . P$ values were corrected for multiple comparisons using the false discovery method (FDR) with a FDR level of 5\% [35]. Transcript levels are presented as a geometric mean and standard error [36]. For ease of presentation, group data, including average, upper, and lower bounds, were then converted back to standard units by raising 2 to the power of the calculated value to obtain fold change values.

\section{Results}

3.1. Demographic Data for Patients with Sleep Apnea. We evaluated 14 patients with sleep apnea (11 male and 3 female) and 8 control subjects (5 male and 3 female) for salivary transcriptional changes associated with sleep apnea. Control subjects did not have any prior existing sleep or mental disorders, were not on prescription medications, had not consumed caffeine, and had not eaten 1 hour prior to providing a saliva sample. Subject age was not different between the two groups (Table 1). The subjects with sleep apnea had an average AHI of $48.5 \pm 23.6$ (mean \pm S.D.). The subjects with sleep apnea had a significantly higher body mass index (BMI) than the controls. Consistent with previous results, subjects with sleep apnea also reported a higher score on the Epworth Sleepiness Scale.

\subsection{Evaluation of Salivary Biomarkers Associated with Inflam-} mation in Patients with Sleep Apnea. We evaluated salivary transcript levels from subjects with sleep apnea and control subjects using low density arrays. The LDA platform simultaneously evaluates the levels of 96 RNA species. Of the 96 genes tested, 21 transcripts were detected in 11 or more sleep apnea patients. Fold changes from control and $P$ value for each gene are presented in Table 2. The $P$ value listed was determined using a two-tailed Student's $t$-test comparing the controls to sleep apnea for all analyzed genes present in saliva. We performed a correction for multiple comparisons using a FDR set at $5 \%$, which yielded a new $P$ value equivalent to 
TABLE 2: Salivary transcript levels in patients with sleep apnea compared to controls.

\begin{tabular}{|c|c|c|c|}
\hline Gene & Abbreviation & $\begin{array}{l}\text { Sleep apnea fold } \Delta \text { from } \\
\text { control }^{\mathrm{a}}\end{array}$ & $P$ value \\
\hline$\beta$-actin & ACTB & $1.43 \pm 0.38$ & 0.191 \\
\hline Arachidonate 5-lipoxygenase & ALOX5 & $1.39 \pm 0.36$ & 0.238 \\
\hline Arachidonate 12-lipoxygenase & ALOX12 & $1.56 \pm 0.69$ & 0.404 \\
\hline Annexin A1 & ANXA1 & $4.62 \pm 1.15$ & $0.003^{\mathrm{b}}$ \\
\hline Annexin A3 & ANXA3 & $1.26 \pm 0.38$ & 0.444 \\
\hline Annexin A5 & ANXA5 & $2.05 \pm 0.92$ & 0.168 \\
\hline$\beta$-2-microglobulin & $\mathrm{B} 2 \mathrm{M}$ & $2.65 \pm 0.70$ & $0.007^{\mathrm{b}}$ \\
\hline Caspase 1, apoptosis-related cysteine peptidase & CASP1 & $2.57 \pm 0.81$ & 0.025 \\
\hline Glyceraldehyde-3-phosphate dehydrogenase & GAPDH & $2.66 \pm 0.81$ & 0.019 \\
\hline Intercellular adhesion molecule 1 & ICAM1 & $0.44 \pm 0.13$ & 0.123 \\
\hline Interleukin 1 receptor, type II & IL1R2 & $1.62 \pm 0.41$ & 0.143 \\
\hline Interleukin 2 receptor, gamma & IL2RG & $2.36 \pm 0.72$ & 0.054 \\
\hline Integrin, alpha $\mathrm{M}$ & ITGAM & $1.50 \pm 0.61$ & 0.376 \\
\hline Integrin, beta 2 & ITGB2 & $1.47 \pm 0.31$ & 0.167 \\
\hline Mitogen-activated protein kinase 1 & MAPK1 & $1.72 \pm 0.66$ & 0.401 \\
\hline Mitogen-activated protein kinase 3 & MAPK3 & $1.30 \pm 0.41$ & 0.585 \\
\hline Mitogen-activated protein kinase 14 & MAPK14 & $1.85 \pm 0.53$ & 0.089 \\
\hline Phosphodiesterase 4B, cAMP-specific & PDE4B & $1.49 \pm 0.21$ & 0.168 \\
\hline $\begin{array}{l}\text { Prostaglandin-endoperoxide synthase } 2 \text { (prostaglandin } \\
\text { G/H synthase and cyclooxygenase) }\end{array}$ & PTGS2 & $3.73 \pm 0.98$ & $0.002^{\mathrm{b}}$ \\
\hline Tumor necrosis factor receptor superfamily, member $1 \mathrm{~A}$ & TNFRSF1A & $1.32 \pm 0.20$ & 0.206 \\
\hline Tumor necrosis factor receptor superfamily, member $1 \mathrm{~B}$ & TNFRSF1B & $1.07 \pm 0.44$ & 0.900 \\
\hline
\end{tabular}

${ }^{\mathrm{a}}$ Mean \pm SEM.

${ }^{\mathrm{b}}$ Significant difference using $5 \%$ FDR post hoc test.

$\alpha=0.0071$ [35]. Compared to controls, 3 transcripts exhibited significant changes in patients with sleep apnea (Table 2), ANXA1, $\beta 2 M$, and PTGS2 (see Table 2 for abbreviations). These results indicate that several inflammatory transcripts are increased in patients with sleep apnea.

3.3. Demographic Data for Patients with an Elevated ESS but without Sleep Apnea. To assess whether the increased inflammatory markers were due primarily to hypoxia or whether they might be more closely associated with increased sleepiness, we evaluated transcriptional changes in an independent group of people that entered the sleep lab suspected of having sleep apnea due to initial screening but ultimately had a normal AHI as determined by PSG (sleepy). The "sleepy" group was composed of 6 males and 12 females. By definition, all individuals in this group had an AHI less than 5. However, all subjects reported ESS scores significantly higher than controls and similar to the subjects with sleep apnea (Tables 1 and 3). "Sleepy" subjects had a similar age to controls but an increased BMI.

3.4. Salivary Transcript Levels in "Sleepy" People. Twentyone transcripts were detected in the saliva of 14 or more "sleepy" patients. As above, to facilitate comparisons with the apnea patients reported in Table 2, Table 4 includes
TABle 3: Demographics for "sleepy" subjects and controls.

\begin{tabular}{lcc}
\hline & Controls $(n=8)$ & Sleepy $(n=18)$ \\
\hline Gender & $5 \mathrm{M}, 3 \mathrm{~F}$ & $6 \mathrm{M}, 12 \mathrm{~F}$ \\
Age & $40.8 \pm 8.9$ & $44.1 \pm 14.4$ \\
AHI & - & $4.7 \pm 5.2$ \\
BMI $^{*}$ & $25.7 \pm 4.4$ & $34.2 \pm 10.8$ \\
ESS $^{*}$ & $3.9 \pm 2.7$ & $11.0 \pm 5.0$ \\
\hline
\end{tabular}

Ave \pm Std. Dev.; ${ }^{*} P<0.05$.

transcripts for fold changes for each of the 21 transcripts. After and FDR correction for multiple comparisons $(\alpha=$ $0.005), 2$ transcripts were significantly changed in the "sleepy" population, CASP1 and PTGS2. PTGS2 can be considered an independent replicate of the apnea results and may deserve further consideration. Thus, inflammatory transcripts are elevated under conditions of EDS.

3.5. Transcriptional Changes Are Not due to Elevated BMI. One possible explanation for elevated inflammatory transcripts is that both the sleep apnea and "sleepy" patients have a significantly higher BMI compared to controls. If the observed relationship is due solely to BMI, then patients with high BMI should have increased levels of inflammatory transcripts compared to patients with lower BMI. Similarly, we 
TABLE 4: Salivary transcript levels in "sleepy" patients compared to controls.

\begin{tabular}{|c|c|c|c|}
\hline Gene & Abbreviation & $\begin{array}{l}\text { Sleepy people fold } \Delta \\
\text { from control }^{\mathrm{a}}\end{array}$ & $P$ value \\
\hline$\beta$-actin & ACTB & $1.86 \pm 0.47$ & 0.033 \\
\hline Arachidonate 5-lipoxygenase & ALOX5 & $1.30 \pm 0.46$ & 0.472 \\
\hline Arachidonate 12-lipoxygenase & ALOX12 & $0.53 \pm 0.33$ & 0.408 \\
\hline Annexin A1 & ANXA1 & $2.86 \pm 1.37$ & 0.099 \\
\hline Annexin A3 & ANXA3 & $2.64 \pm 0.85$ & 0.010 \\
\hline Annexin A5 & ANXA5 & $2.29 \pm 0.76$ & 0.054 \\
\hline$\beta$-2-microglobulin & B2M & $3.33 \pm 1.34$ & 0.013 \\
\hline Caspase 1, apoptosis-related cysteine peptidase & CASP1 & $5.36 \pm 2.07$ & $0.001^{\mathrm{b}}$ \\
\hline Glyceraldehyde-3-phosphate dehydrogenase & GAPDH & $2.35 \pm 0.88$ & 0.068 \\
\hline Intercellular adhesion molecule 1 & ICAM1 & $0.61 \pm 0.33$ & 0.441 \\
\hline Interleukin 1 receptor, type II & IL1R2 & $2.04 \pm 0.75$ & 0.092 \\
\hline Interleukin 2 receptor, gamma & IL2RG & $3.53 \pm 2.25$ & 0.118 \\
\hline Integrin, alpha $\mathrm{M}$ & ITGAM & $1.64 \pm 0.65$ & 0.292 \\
\hline Integrin, beta 2 & ITGB2 & $1.84 \pm 0.52$ & 0.102 \\
\hline Mitogen-activated protein kinase 1 & MAPK1 & $1.55 \pm 0.65$ & 0.510 \\
\hline Mitogen-activated protein kinase 3 & MAPK3 & $1.06 \pm 0.42$ & 0.921 \\
\hline Mitogen-activated protein kinase 14 & MAPK14 & $2.33 \pm 0.77$ & 0.057 \\
\hline Phosphodiesterase 4B, cAMP-specific & PDE4B & $1.69 \pm 0.69$ & 0.259 \\
\hline $\begin{array}{l}\text { Prostaglandin-endoperoxide synthase } 2 \text { (prostaglandin } \\
\text { G/H synthase and cyclooxygenase) }\end{array}$ & PTGS2 & $3.80 \pm 1.34$ & $0.005^{\mathrm{b}}$ \\
\hline Tumor necrosis factor receptor superfamily, member $1 \mathrm{~A}$ & TNFRSF1A & $1.87 \pm 0.62$ & 0.096 \\
\hline Tumor necrosis factor receptor superfamily, member $1 \mathrm{~B}$ & TNFRSF1B & $1.80 \pm 0.88$ & 0.322 \\
\hline
\end{tabular}

${ }^{\mathrm{a}}$ Mean \pm SEM.

${ }^{\mathrm{b}}$ Significant difference using 5\% FDR post hoc test.

should detect a significant positive correlation between BMI and the level of salivary transcripts. To test this hypothesis we discretized the data by placing the subjects with a low BMI $(\leq 30 ; n=24)$ into a single group and comparing them to subjects with a high BMI $(>38 ; n=16)$. As seen in Table 5 , transcripts were not significantly different between subjects in the lower BMI group compared to their counterparts with a higher BMI. In addition, we evaluated the relationship between BMI and transcript levels using a Pearson correlation and found no significant correlations. Examples of transcript expression levels for the genes with the lowest $P$ values are plotted in Figure 1 as a function of BMI. These data indicate that, in this dataset, BMI does not account for increases in transcript levels in these patients.

\section{Discussion}

In this paper, we report that salivary transcript levels of inflammatory genes are increased both in patients with sleep apnea and in an independent cohort of patients who self-identified as sleepy and were referred to the sleep lab suspected of having sleep apnea. We have previously used these same human low density arrays as a discovery tool to identify genes that are modified following acute sleep deprivation in healthy adults [24]. In that study, we reported that integrin, $\alpha M$ (ITGAM), and annexin A3 (ANXA3) were significantly increased following $24 \mathrm{~h}$ and $30 \mathrm{~h}$ of waking. Interestingly, neither ITGAM nor ANXA3 were altered in either sleep apnea or "sleepy" subjects suggesting that they may be better suited for detecting acute sleep loss than for identifying patients with chronic sleep disruption. In contrast, salivary PTGS2 was significantly increased in both sleep apnea patients and in the "sleepy" cohort but was not affected by acute sleep deprivation [24]. Thus, these data support our previous proposal that a panel of biomarkers will be required to accurately identify sleep deprived individuals and further suggest that different sets of biomarkers may be required to distinguish between acute and chronic sleep disruptions.

Sleep apnea has been particularly difficult to diagnose and treat and the incidence of sleep apnea has been difficult to determine. Estimates of sleep apnea have ranged from as low as $2-4 \%$ to as high as $24 \%$ of middle aged men [37], although other studies have estimated the likelihood of sleep apnea to be much higher still $[38,39]$. Moreover, it has been suggested that sleep apnea is likely to be substantially underdiagnosed $[38,39]$. Indeed, years may transpire between the onset of sleep apnea and the eventual diagnosis [40]. As the population ages and becomes more obese, the prevalence of sleep apnea is expected to increase even further [7, 41, 42]. During these intervening years, the severity and consequences of the sleep apnea may also increase [41, 42]. The associated 
TABLE 5: Comparison of transcriptional changes in high and low BMI subjects.

\begin{tabular}{|c|c|c|c|c|}
\hline Gene & Abbreviation & $\begin{array}{l}\mathrm{BMI}<30 \\
\text { fold } \Delta^{\mathrm{a}, \mathrm{b}}\end{array}$ & $\begin{array}{l}\text { BMI }>30 \\
\text { fold } \Delta^{\mathrm{a}, \mathrm{c}}\end{array}$ & $t$-test \\
\hline$\beta$-actin & ACTB & $1.39 \pm 0.29$ & $1.65 \pm 0.40$ & 0.438 \\
\hline Arachidonate 5-lipoxygenase & ALOX5 & $1.46 \pm 0.33$ & $0.97 \pm 0.28$ & 0.117 \\
\hline Arachidonate 12-lipoxygenase & ALOX12 & $1.25 \pm 0.47$ & $0.48 \pm 0.32$ & 0.058 \\
\hline Annexin A1 & ANXA1 & $3.06 \pm 1.25$ & $2.33 \pm 1.03$ & 0.529 \\
\hline Annexin A3 & ANXA3 & $1.68 \pm 0.40$ & $1.63 \pm 0.61$ & 0.913 \\
\hline Annexin A5 & ANXA5 & $1.74 \pm 0.58$ & $2.17 \pm 0.75$ & 0.519 \\
\hline$\beta$-2-microglobulin & B2M & $1.95 \pm 0.66$ & $3.12 \pm 0.91$ & 0.156 \\
\hline Caspase 1, apoptosis-related cysteine peptidase & CASP1 & $2.68 \pm 0.90$ & $3.05 \pm 1.42$ & 0.741 \\
\hline Glyceraldehyde-3-phosphate dehydrogenase & GAPDH & $2.14 \pm 0.72$ & $1.94 \pm 0.57$ & 0.771 \\
\hline Intercellular adhesion molecule 1 & ICAM1 & $0.61 \pm 0.22$ & $0.62 \pm 0.36$ & 0.982 \\
\hline Interleukin 1 receptor, type II & IL1R2 & $1.88 \pm 0.53$ & $1.24 \pm 0.34$ & 0.153 \\
\hline Interleukin 2 receptor, gamma & IL2RG & $2.65 \pm 1.37$ & $2.20 \pm 0.93$ & 0.713 \\
\hline Integrin, alpha $\mathrm{M}$ & ITGAM & $1.56 \pm 0.48$ & $1.25 \pm 0.51$ & 0.537 \\
\hline Integrin, beta 2 & ITGB2 & $1.52 \pm 0.35$ & $1.54 \pm 0.38$ & 0.949 \\
\hline Mitogen-activated protein kinase 1 & MAPK1 & $1.77 \pm 0.83$ & $1.20 \pm 0.37$ & 0.355 \\
\hline Mitogen-activated protein kinase 3 & MAPK3 & $1.41 \pm 0.36$ & $0.90 \pm 0.34$ & 0.156 \\
\hline Mitogen-activated protein kinase 14 & MAPK14 & $2.18 \pm 0.60$ & $1.51 \pm 0.45$ & 0.211 \\
\hline Phosphodiesterase 4B, cAMP-specific & PDE4B & $1.44 \pm 0.36$ & $1.44 \pm 0.55$ & 0.999 \\
\hline $\begin{array}{l}\text { Prostaglandin-endoperoxide synthase } 2 \text { (prostaglandin } \\
\text { G/H synthase and cyclooxygenase) }\end{array}$ & PTGS2 & $2.43 \pm 0.84$ & $3.42 \pm 1.13$ & 0.338 \\
\hline Tumor necrosis factor receptor superfamily, member $1 \mathrm{~A}$ & TNFRSF1A & $1.47 \pm 0.36$ & $1.40 \pm 0.33$ & 0.841 \\
\hline Tumor necrosis factor receptor superfamily, member $1 \mathrm{~B}$ & TNFRSF1B & $1.72 \pm 0.66$ & $0.86 \pm 0.36$ & 0.099 \\
\hline
\end{tabular}

${ }^{\mathrm{a}}$ Mean \pm SEM.

${ }^{\mathrm{b}} n=15-24$.

${ }^{c} n=11-15$.

consequences of sleep apnea include increased daytime sleepiness [3] and an increased likelihood of automobile accidents [6]. Moreover, sleep apnea has been associated with a host of cardiovascular complications $[43,44]$ and increased rates of all-cause mortality [7]. Sleep apnea has also been associated with learning impairments [45]. The current gold standard of treatment, continuous positive airway pressure (CPAP), may be able to reverse some of the consequences of sleep apnea, such as sleepiness [2] and restoration of typical sleep stages [2], and may lower blood pressure [46] but the literature is less conclusive whether cognitive function is improved [4, 47]. Thus, a clinical goal is to have patients begin treatment for sleep apnea as soon as possible.

A simple cost-effective biomarker that can be easily used at the point of care may be a useful tool to convince people to go to the sleep lab for a more precise diagnosis and treatment. Since biomarkers are both objective and quantifiable, they are well suited for persuading an individual that they may have an underlying affliction that needs greater attention. In that regard, it is important to note that the well-known relationship between inflammation and sleep deprivation is an advantage since many physicians are likely to be familiar with this relationship and thus more willing to incorporate such a biomarker into their treatment practices. That is, numerous inflammatory markers, including IL-6 [20, 26, 48],
IL-8 [27, 28, 48], TNF- $\alpha$ [26], CRP [49, 50], monocyte chemoattractant protein-1 (MCP-1) [27], ICAM [27, 51], selectins [51], VCAM [51], nitric oxide [52], and isoprostane [20], are elevated in body fluids of patients with sleep apnea. Moreover, proteomics have identified protein biomarkers in urine [53]. Interestingly, inflammatory markers have also been associated with sleep deprivation in serum $[54,55]$ and saliva in healthy adults [24]. In this study, transcript levels for PTGS2 were elevated in people with inadequate sleep. Our results parallel findings in mice in which transcript levels for PTGS2 are elevated after acute sleep deprivation [56]. PTGS2, along with PTGS1, is a critical enzyme in prostaglandin synthesis. Prostaglandins initiate a set of molecular cascades that result in an inflammatory response. PTGS2 is induced by several stimuli including proinflammatory signals and converts arachidonic acid to prostaglandin $\mathrm{H} 2$ [57]. Thus, PTGS2 plays a role in the inflammatory pathway in addition to being a candidate biomarker for EDS.

It is interesting to note that many studies compare sleep apnea patients to healthy controls. Although this approach has been quite successful, our data suggest that other patients with chronic sleep disruption may also experience some of the same outcomes as patients with sleep apnea. If this turns out to be the case, more generally, assessing chronically sleepy individuals along with patients that have sleep apnea 

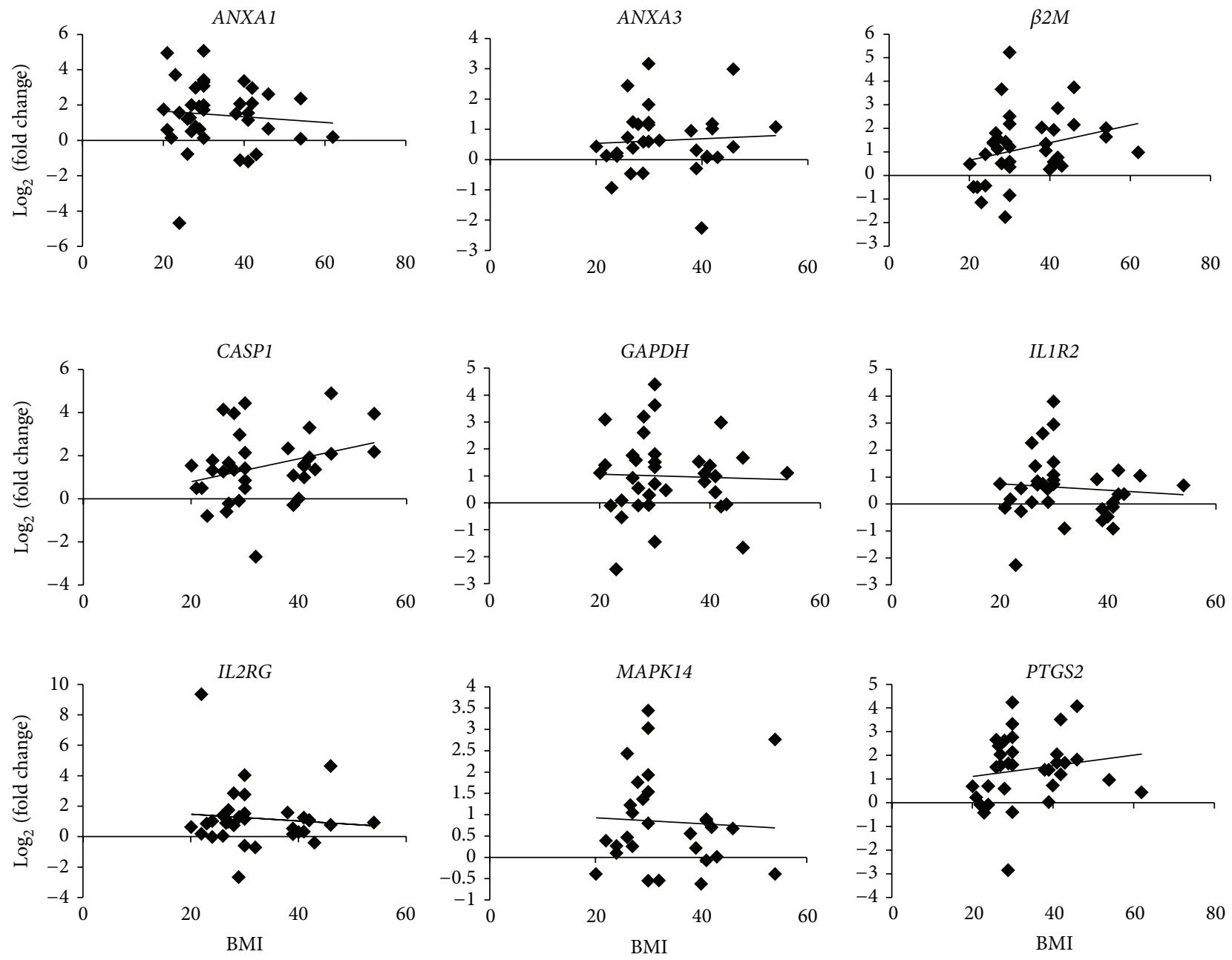

Figure 1: Correlation of transcript levels with BMI. The transcript levels were plotted against the log values of the transcript levels for each of the transcripts that were significant in the sleep apnea or "sleepy" groups. In addition the transcripts with the lowest $P$ value were plotted to determine if there was a significant correlation with increased weight. For each transcript, there was no significant correlation with increased BMI.

may facilitate the identification of precise deficits that are due specifically to sleep loss rather than other features of sleep apnea, such as hypoxia [48]. Nonetheless, our results emphasize that PTGS2 cannot distinguish between sleepy patients with and without sleep apnea. While this may seem disappointing, we believe that any diagnosis of a sleep disorder should be made by a qualified sleep physician after fully examining the patient. Indeed, it may not be possible to identify a biomarker that is specific to a single sleep disorder. One example of a promising biomarker is cerebrospinal fluid (CSF) levels of hypocretin-1/orexin (HCRT), which has emerged from research in the narcolepsy field $[18,58]$. CSF levels of hypocretin-1/orexin are lower in narcoleptic patients. But for patients without cataplexy, decreased CSF levels of HCRT are not as predictive, even in combination with the genetic marker DBQ. Moreover, decreases in CSF levels of HCRT are also associated now, being associated with other diseases, including dementia with Lewy Body [59] and Alzheimer's disease [60] and Kleine-Levin syndrome [58, 61]. This example illustrates the difficulty of a single biomarker with a given sleep disorder. Nonetheless, a biomarker that can help primary physicians to identify sleepy individuals may be extremely useful for ensuring that they get the appropriate care in a timely manner.

Our protocol has two aspects that may be useful for the real-world application of biomarkers to identify patients with sleep disorders. First, acquiring saliva is a noninvasive procedure and does not, for example, require a private location for sample collection (e.g., urine) or specialized skills to acquire samples (e.g., blood). As a consequence, salivary biomarkers can be readily used both on the roadside and in a doctor's office with equal effectiveness. A second advantage of our protocol is our focus on salivary RNA coupled with the LDA platform for the rapid detection of 96 transcripts simultaneously. LDAs may be an effective way to screen multiple targeted transcripts at once without the burden that NextGen sequencing frequently demands. One advantage of using transcript levels as the biomarker is that nucleotide probes can be generated quickly and are very specific for the target intended as opposed to having to design an antibody 
against a protein target. Given human variability, the different roles that genes can play throughout the body (pleiotropy), and the likelihood that a given analyte will be induced by a variety of environmental situations, it is likely that no single biomarker will be ideal for reliable diagnosis of sleep apnea. Nonetheless, our data suggest that PTGS2 may be a particularly good candidate for inclusion in a panel of biomarkers.

There are two potential weaknesses of our study. The first is the sample size of our groups. Low sample sizes may identify significant differences within the subgroup that does not generalize to the rest of the population. In this study, we have tried to balance the difficulty of completing discovery experiments with the expense of a human study. Although our sample size was low for sleep apnea subjects, we were able to confirm the changes in PTGS2 in a separate cohort of "sleepy" patients. This confirmation adds to our confidence that PTGS2 is a good candidate for followup studies. A second weakness of this study is that we were not able to control for the levels of BMI between our control subjects and patients with sleep apnea or the "sleepy" cohort. To address this issue, we discretized the subjects into groups with low or high BMI. Means testing between the two groups indicated the subjects with low and high BMI were not different from one another. Moreover, Pearson correlation between BMI and transcript levels did not reveal any significant associations. Finally, when examined as a whole, we could find no general trend of inflammatory genes being elevated in subjects with higher BMI. Thus, while BMI is a potential confounder, it did not appear to unduly influence the results of this study.

Biomarkers have the capacity to serve an important need in maintaining human health. They provide an objective insight into the goings-on of the body. In particular, there is a need to identify individuals with sleep problems that result in EDS. Given the difficulty in identifying individuals, the consequences of sleep apnea and other sleep disorders, and the potential that therapy possesses, objective biomarkers can be a way to convince people to go to the sleep lab for diagnosis. We have demonstrated the feasibility of using saliva to identify changes in inflammatory transcripts that correlate with the presence of EDS. Saliva is an ideal source of biofluid for a potential source of biomarkers. Obtaining saliva is a noninvasive and relatively inexpensive process. Further experiments in larger and independent populations will determine the precision and validity of these biomarkers, but these experiments demonstrate that saliva has the potential to be objective biomarkers to identify subjects with EDS.

\section{Conflict of Interests}

The authors declare that there is no conflict of interests regarding the publication of this paper.

\section{Acknowledgments}

The authors thank Laura Gottschalk for technical assistance and all of the subjects for agreeing to participate in this study. This work was supported by NIMH HL092731.

\section{References}

[1] H. P. A. van Dongen, G. Maislin, J. M. Mullington, and D. F. Dinges, "The cumulative cost of additional wakefulness: dose-response effects on neurobehavioral functions and sleep physiology from chronic sleep restriction and total sleep deprivation," Sleep, vol. 26, no. 2, pp. 117-126, 2003.

[2] J. Lamphere, T. Roehrs, R. Wittig, F. Zorick, W. A. Conway, and T. Roth, "Recovery of alertness after CPAP in apnea," Chest, vol. 96, no. 6, pp. 1364-1367, 1989.

[3] M. W. Johns, "A new method for measuring daytime sleepiness: the Epworth sleepiness scale," Sleep, vol. 14, no. 6, pp. 540-545, 1991.

[4] L. Ferini-Strambi, C. Baietto, M. R. di Gioia et al., "Cognitive dysfunction in patients with obstructive sleep apnea (OSA): partial reversibility after continuous positive airway pressure (CPAP)," Brain Research Bulletin, vol. 61, no. 1, pp. 87-92, 2003.

[5] G. K. Zammit, V. N. Joish, M. C. Kong, R. Balkrishnan, D. Lerner, and M. Rosekind, "Impact of nighttime awakenings on worker productivity and performance," Journal of Occupational \& Environmental Medicine, vol. 52, no. 5, pp. 513-518, 2010.

[6] J. Teran-Santos, A. Jimenez-Gomez, J. Cordero-Guevara, and Cooperative Group Burgos-Santander, "The association between sleep apnea and the risk of traffic accidents," The New England Journal of Medicine, vol. 340, no. 11, pp. 847-851, 1999.

[7] T. Young, L. Finn, P. E. Peppard et al., "Sleep disordered breathing and mortality: eighteen-year follow-up of the Wisconsin sleep cohort," Sleep, vol. 31, no. 8, pp. 1071-1078, 2008.

[8] A. M. Ingiosi, M. R. Opp, and J. M. Krueger, "Sleep and immune function: glial contributions and consequences of aging," Current Opinion in Neurobiology, vol. 23, no. 5, pp. 806811, 2013.

[9] R. Nadeem, J. Molnar, E. M. Madbouly et al., "Serum inflammatory markers in obstructive sleep apnea: a meta-analysis," Journal of Clinical Sleep Medicine, vol. 9, pp. 1003-1012, 2013.

[10] J. K. Cremeans-Smith, K. Millington, E. Sledjeski, K. Greene, and D. L. Delahanty, "Sleep disruptions mediate the relationship between early postoperative pain and later functioning following total knee replacement surgery," Journal of Behavioral Medicine, vol. 29, no. 2, pp. 215-222, 2006.

[11] E. Vanninen, A. Tuunainen, M. Kansanen, M. Uusitupa, and E. Länsimies, "Cardiac sympathovagal balance during sleep apnea episodes," Clinical Physiology, vol. 16, no. 3, pp. 209-216, 1996.

[12] E. Tasali, R. Leproult, D. A. Ehrmann, and E. van Cauter, "Slow-wave sleep and the risk of type 2 diabetes in humans," Proceedings of the National Academy of Sciences of the United States of America, vol. 105, no. 3, pp. 1044-1049, 2008.

[13] M. W. Johns, A. Tucker, R. Chapman, K. Crowley, and N. Michael, "Monitoring eye and eyelid movements by infrared reflectance oculography to measure drowsiness in drivers," Somnologie, vol. 11, no. 4, pp. 234-242, 2007.

[14] A. Tietäväinen, F. K. Gates, A. Meriläinen, J. E. Mandel, and E. Hæggström, "Nintendo Wii Fit based sleepiness tester detects impairment of postural steadiness due to $24 \mathrm{~h}$ of wakefulness," Medical Engineering and Physics, vol. 35, no. 12, pp. 1850-1853, 2013.

[15] N. Naidoo, "Potential of proteomics as a bioanalytic technique for quantifying sleepiness," Journal of Clinical Sleep Medicine, vol. 7, no. 5, pp. S28-S30, 2011.

[16] M. Sánchez-de-la-Torre, A. Barceló, J. Piérola et al., "Plasma levels of neuropeptides and metabolic hormones, and sleepiness 
in obstructive sleep apnea," Respiratory Medicine, vol. 105, no. 12, pp. 1954-1960, 2011.

[17] S. Nishino, B. Ripley, S. Overeem, G. J. Lammers, and E. Mignot, "Hypocretin (orexin) deficiency in human narcolepsy," The Lancet, vol. 355, no. 9197, pp. 39-40, 2000.

[18] E. Mignot, G. J. Lammers, B. Ripley et al., "The role of cerebrospinal fluid hypocretin measurement in the diagnosis of narcolepsy and other hypersomnias," Archives of Neurology, vol. 59, no. 10, pp. 1553-1562, 2002.

[19] O. Andlauer, H. Moore IV, S.-C. Hong et al., "Predictors of hypocretin (orexin) deficiency in narcolepsy without cataplexy," Sleep, vol. 35, no. 9, pp. 1247-1255, 2012.

[20] G. E. Carpagnano, S. A. Kharitonov, O. Resta, M. P. FoschinoBarbaro, E. Gramiccioni, and P. J. Barnes, "Increased 8isoprostane and interleukin- 6 in breath condensate of obstructive sleep apnea patients," Chest, vol. 122, no. 4, pp. 1162-1167, 2002.

[21] L. Seugnet, J. Boero, L. Gottschalk, S. P. Duntley, and P. J. Shaw, "Identification of a biomarker for sleep drive in flies and humans," Proceedings of the National Academy of Sciences of the United States of America, vol. 103, no. 52, pp. 19913-19918, 2006.

[22] L. Seugnet, Y. Suzuki, M. Thimgan et al., "Identifying sleep regulatory genes using a Drosophila model of insomnia," The Journal of Neuroscience, vol. 29, no. 22, pp. 7148-7157, 2009.

[23] M. S. Thimgan, S. P. Duntley, and P. J. Shaw, "Changes in gene expression with sleep," Journal of Clinical Sleep Medicine, vol. 7, no. 5, pp. S26-S27, 2011.

[24] M. S. Thimgan, L. Gottschalk, C. Toedebusch et al., "Crosstranslational studies in human and Drosophila identify markers of sleep loss," PLoS ONE, vol. 8, no. 4, Article ID e61016, 2013.

[25] V. Bachmann, F. Klaus, S. Bodenmann et al., "Functional ADA polymorphism increases sleep depth and reduces vigilant attention in humans," Cerebral Cortex, vol. 22, no. 4, pp. 962970, 2012.

[26] A. N. Vgontzas, D. A. Papanicolaou, E. O. Bixler, A. Kales, K. Tyson, and G. P. Chrousos, "Elevation of plasma cytokines in disorders of excessive daytime sleepiness: role of sleep disturbance and obesity," The Journal of Clinical Endocrinology and Metabolism, vol. 82, no. 5, pp. 1313-1316, 1997.

[27] E. Ohga, T. Tomita, H. Wada, H. Yamamoto, T. Nagase, and Y. Ouchi, "Effects of obstructive sleep apnea on circulating ICAM1, IL-8, and MCP-1," Journal of Applied Physiology, vol. 94, no. 1, pp. 179-184, 2003.

[28] M. A. Alzoghaibi and A. S. O. Bahammam, "Lipid peroxides, superoxide dismutase and circulating IL-8 and GCP-2 in patients with severe obstructive sleep apnea: a pilot study," Sleep and Breathing, vol. 9, no. 3, pp. 119-126, 2005.

[29] T. U. Ciftci, O. Kokturk, N. Bukan, and A. Bilgihan, "The relationship between serum cytokine levels with obesity and obstructive sleep apnea syndrome," Cytokine, vol. 28, no. 2, pp. 87-91, 2004.

[30] L. M. O’Brien, R. Tauman, and D. Gozal, “Sleep pressure correlates of cognitive and behavioral morbidity in snoring children," Sleep, vol. 27, no. 2, pp. 279-282, 2004.

[31] A. Ursavaş, M. Karadağ, E. Rodoplu, A. Yilmaztepe, H. B. Oral, and R. O. Gözü, "Circulating ICAM-1 and VCAM-1 levels in patients with obstructive sleep apnea syndrome," Respiration, vol. 74, no. 5, pp. 525-532, 2007.

[32] K. Chin, T. Nakamura, K. Shimizu, M. Mishima, M. Miyasaka, and M. Ohi, "Effects of nasal continuous positive airway pressure on soluble cell adhesion molecules in patients with obstructive sleep apnea syndrome," American Journal of Medicine, vol. 109, no. 7, pp. 562-567, 2000.

[33] S. P. Duntley, A. H. Kim, D. L. Silbergeld, and J. W. Miller, "Characterization of the mu rhythm during rapid eye movement sleep," Clinical Neurophysiology, vol. 112, no. 3, pp. 528-531, 2001.

[34] K. J. Livak and T. D. Schmittgen, "Analysis of relative gene expression data using real-time quantitative PCR and the $2^{-\triangle \Delta C_{T}}$ method," Methods, vol. 25, no. 4, pp. 402-408, 2001.

[35] Y. Benjamini and Y. Hochberg, "Controlling the false discovery rate: a practical and powerful approach to multiple testing," Journal of the Royal Statistical Society Series B: Methodological, vol. 57, no. 1, pp. 289-300, 1995.

[36] E. F. Alf and J. M. Grossberg, "The geometric mean: confidence limits and significance tests," Perception and Psychophysics, vol. 26, no. 5, pp. 419-421, 1979.

[37] T. Young, M. Palta, J. Dempsey, J. Skatrud, S. Weber, and S. Badr, "The occurrence of sleep-disordered breathing among middleaged adults," The New England Journal of Medicine, vol. 328, no. 17, pp. 1230-1235, 1993.

[38] D. M. Hiestand, P. Britz, M. Goldman, and B. Phillips, "Prevalence of symptoms and risk of sleep apnea in the US population: results from the national sleep foundation sleep in America 2005 poll," Chest, vol. 130, no. 3, pp. 780-786, 2006.

[39] K. J. Finkel, A. C. Searleman, H. Tymkew et al., "Prevalence of undiagnosed obstructive sleep apnea among adult surgical patients in an academic medical center," Sleep Medicine, vol. 10, no. 7, pp. 753-758, 2009.

[40] F. Rahaghi and R. C. Basner, "Delayed diagnosis of obstructive sleep apnea: don't ask, don't tell," Sleep and Breathing, vol. 3, no. 4, pp. 119-124, 1999.

[41] P. E. Peppard, T. Young, M. Palta, J. Dempsey, and J. Skatrud, "Longitudinal study of moderate weight change and sleepdisordered breathing," The Journal of the American Medical Association, vol. 284, no. 23, pp. 3015-3021, 2000.

[42] P. E. Peppard, T. Young, J. H. Barnet, M. Palta, E. W. Hagen, and K. M. Hla, "Increased prevalence of sleep-disordered breathing in adults," The American Journal of Epidemiology, vol. 177, no. 9, pp. 1006-1014, 2013.

[43] P. E. Peppard, T. Young, M. Palta, and J. Skatrud, "Prospective study of the association between sleep-disordered breathing and hypertension," The New England Journal of Medicine, vol. 342, no. 19, pp. 1378-1384, 2000.

[44] T. Young, P. Peppard, M. Palta et al., "Population-based study of sleep-disordered breathing as a risk factor for hypertension," Archives of Internal Medicine, vol. 157, no. 15, pp. 1746-1752, 1997.

[45] K. Cheshire, H. Engleman, I. Deary, C. Shapiro, and N. J. Douglas, "Factors impairing daytime performance in patients with sleep apnea/hypopnea syndrome," Archives of Internal Medicine, vol. 152, no. 3, pp. 538-541, 1992.

[46] M.-Á. Martínez-García, F. Capote, F. Campos-Rodríguez et al., "Effect of CPAP on blood pressure in patients with obstructive sleep apnea and resistant hypertension: the HIPARCO randomized clinical trial," The Journal of the American Medical Association, vol. 310, no. 22, pp. 2407-2415, 2013.

[47] H. M. Engleman, K. E. Cheshire, I. J. Deary, and N. J. Douglas, "Daytime sleepiness, cognitive performance and mood after continuous positive airway pressure for the sleep apnoea/hypopnoea syndrome," Thorax, vol. 48, no. 9, pp. 911914, 1993. 
[48] G. E. Carpagnano, A. Spanevello, R. Sabato et al., "Systemic and airway inflammation in sleep apnea and obesity: the role of ICAM-1 and IL-8," Translational Research, vol. 155, no. 1, pp. 35-43, 2010.

[49] A. S. M. Shamsuzzaman, M. Winnicki, P. Lanfranchi et al., "Elevated C-reactive protein in patients with obstructive sleep apnea," Circulation, vol. 105, no. 21, pp. 2462-2464, 2002.

[50] T. Yokoe, K. Minoguchi, H. Matsuo et al., "Elevated levels of Creactive protein and interleukin-6 in patients with obstructive sleep apnea syndrome are decreased by nasal continuous positive airway pressure," Circulation, vol. 107, no. 8, pp. 11291134, 2003.

[51] E. Ohga, T. Nagase, T. Tomita et al., "Increased levels of circulating ICAM-1, VCAM-1, and L-selectin in obstructive sleep apnea syndrome," Journal of Applied Physiology, vol. 87, no. 1, pp. 10-14, 1999.

[52] G. E. Carpagnano, A. Spanevello, R. Sabato, A. Depalo, V. Turchiarelli, and M. P. Foschino Barbaro, "Exhaled pH, exhaled nitric oxide, and induced sputum cellularity in obese patients with obstructive sleep apnea syndrome," Translational Research, vol. 151, no. 1, pp. 45-50, 2008.

[53] D. Gozal, S. Jortani, A. B. Snow et al., "Two-dimensional differential in-gel electrophoresis proteomic approaches reveal urine candidate biomarkers in pediatric obstructive sleep apnea," American Journal of Respiratory and Critical Care Medicine, vol. 180, no. 12, pp. 1253-1261, 2009.

[54] L. Redwine, R. L. Hauger, J. C. Gillin, and M. Irwin, "Effects of sleep and sleep deprivation on interleukin-6, growth hormone, cortisol, and melatonin levels in humans," Journal of Clinical Endocrinology and Metabolism, vol. 85, no. 10, pp. 3597-3603, 2000.

[55] D. J. Frey, M. Fleshner, and K. P. Wright Jr., "The effects of 40 hours of total sleep deprivation on inflammatory markers in healthy young adults," Brain, Behavior, and Immunity, vol. 21, no. 8, pp. 1050-1057, 2007.

[56] S. Maret, S. Dorsaz, L. Gurcel et al., "Homerla is a core brain molecular correlate of sleep loss," Proceedings of the National Academy of Sciences of the United States of America, vol. 104, no. 50, pp. 20090-20095, 2007.

[57] E. Ricciotti and G. A. Fitzgerald, "Prostaglandins and inflammation," Arteriosclerosis, Thrombosis, and Vascular Biology, vol. 31, no. 5, pp. 986-1000, 2011.

[58] Y. Dauvilliers, C. R. Baumann, B. Carlander et al., "CSF hypocretin-1 levels in narcolepsy, Kleine-Levin syndrome, and other hypersomnias and neurological conditions," Journal of Neurology, Neurosurgery and Psychiatry, vol. 74, no. 12, pp. 1667$1673,2003$.

[59] M. Wennström, E. Londos, L. Minthon, and H. M. Nielsen, "Altered CSF orexin and alpha-synuclein levels in dementia patients," Journal of Alzheimer's Disease, vol. 29, no. 1, pp. 125132, 2012.

[60] R. Fronczek, S. van Geest, M. Frölich et al., "Hypocretin (orexin) loss in Alzheimer's disease," Neurobiology of Aging, vol. 33, no. 8, pp. 1642-1650, 2012.

[61] R. Lopez, L. Barateau, S. Chenini, and Y. Dauvilliers, "Preliminary results on CSF biomarkers for hypothalamic dysfunction in Kleine-Levin syndrome," Sleep Medicine, vol. 16, no. 1, pp. 194-196, 2015. 


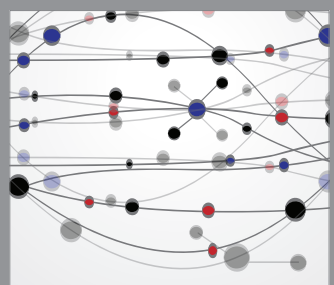

The Scientific World Journal
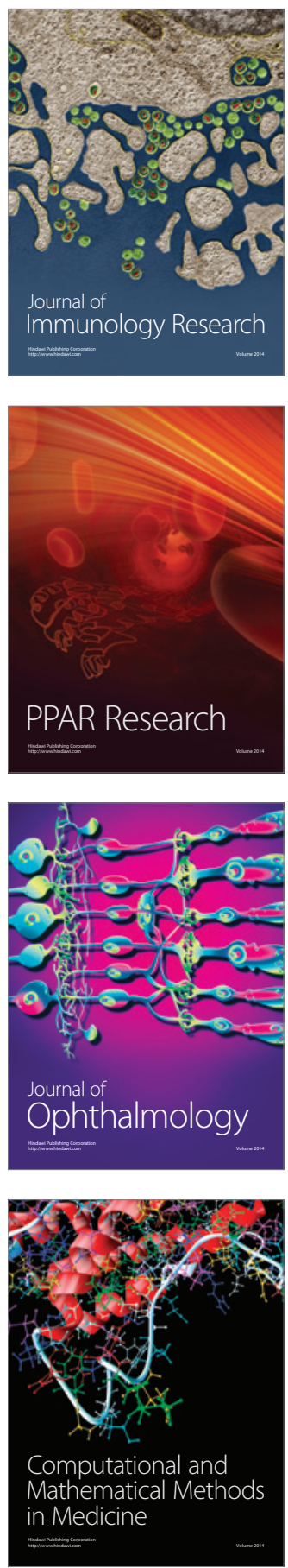

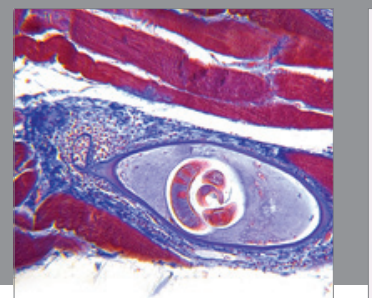

Gastroenterology

Research and Practice
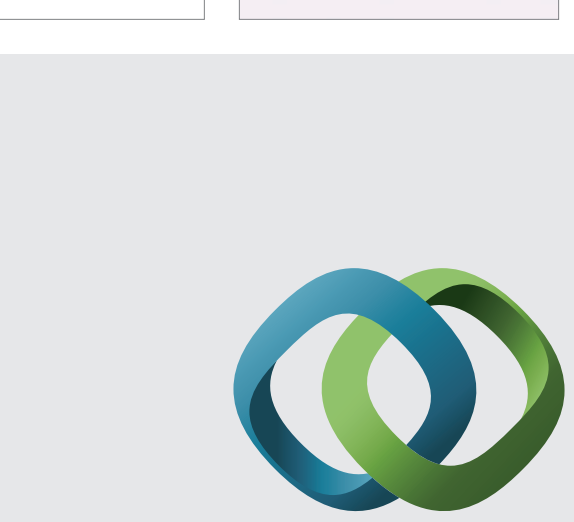

\section{Hindawi}

Submit your manuscripts at

http://www.hindawi.com
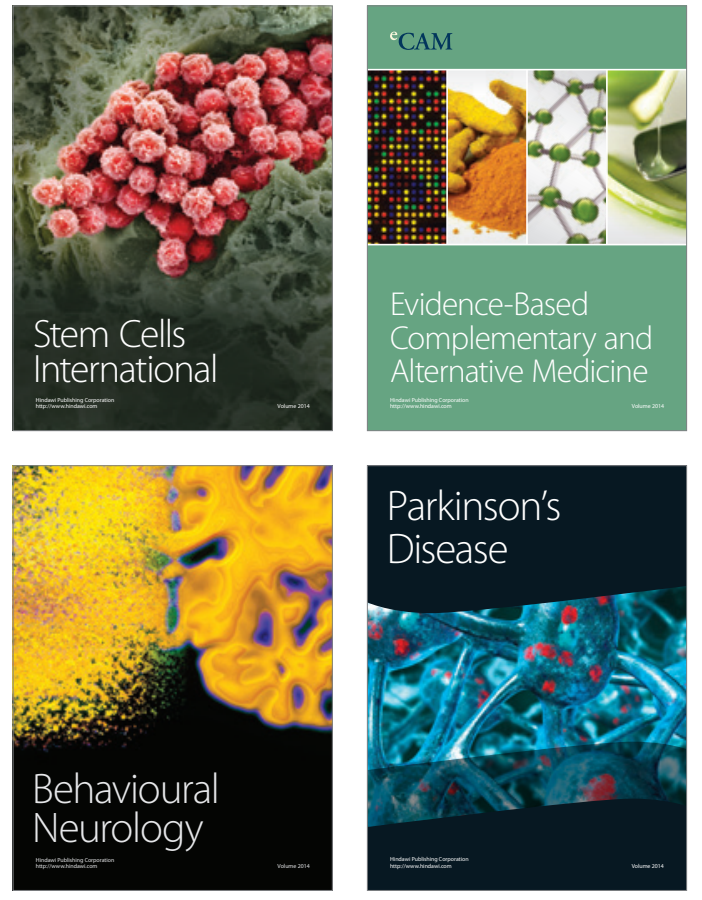
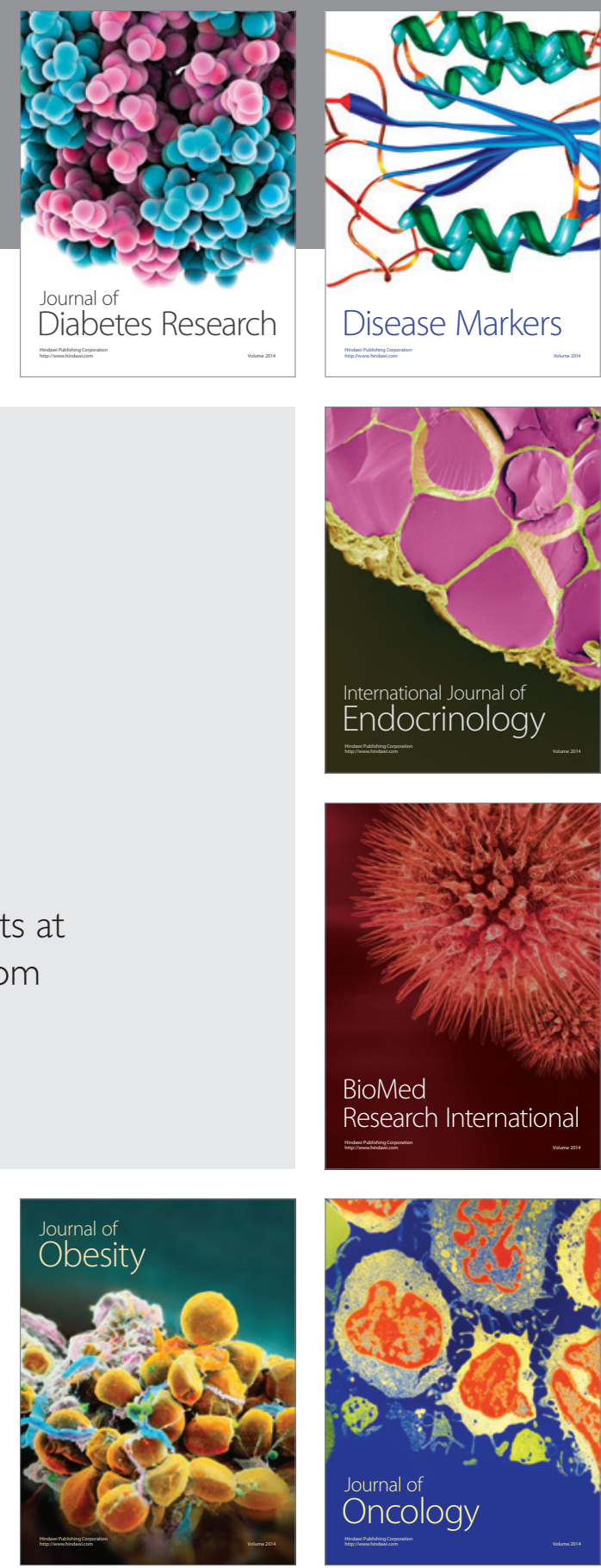

Disease Markers
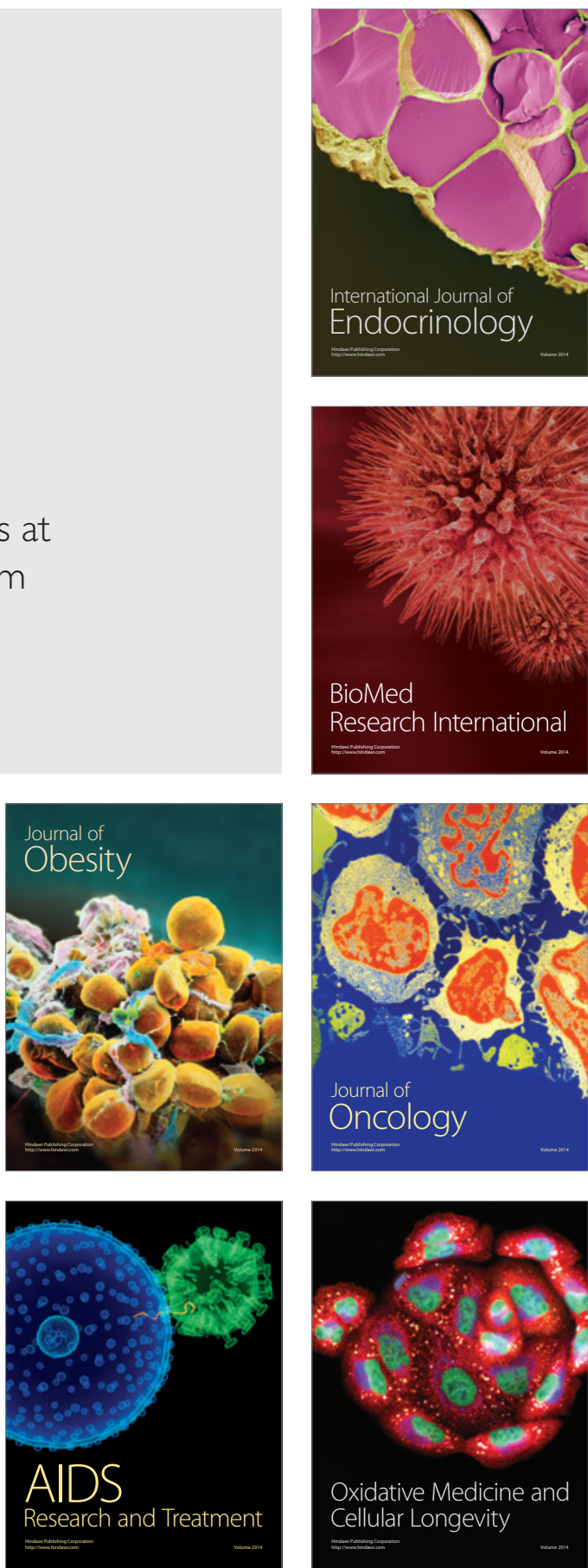